\title{
Embedded unit for point-of-care impedance based biosensor readout
}

\author{
Jeroen Broeders, Dieter Croux, Ans Weustenraed, \\ Thomas Cleij, Patrick Wagner and Ward De \\ Ceuninck \\ Institute for Materials Research \\ Hasselt University \\ Diepenbeek, Belgium \\ Jeroen.broeders@uhasselt.be
}

\author{
Wouter Vanaken, Stijn Duchateau and Ronald \\ Thoelen \\ Xios University College \\ Diepenbeek, Belgium \\ Ronald.thoelen@xios.be
}

\begin{abstract}
Bridging the gap between state of the art consumer electronics and bio-analytical setups designed for lab environments, an embedded, miniaturised, stand-alone measurement device is developed. The presented impedance analyser incorporates most of the data communication features present in current-generation smartphones and is specifically optimised for low-frequency impedance based biosensor readout. The compact unit operates fully stand-alone with a touchscreen. Its functionality was tested and verified. In this work the unit is tested by using a biomimetic sensing device for the detection of L-nicotine. The combination between the handheld, embedded design of this specialised measurement equipment and a sensor layout fine-tuned for specific applications could mean significant advances in point-of-care systems.
\end{abstract} alone

Keywords- impedance, embedded systems, biosensors, stand-

\section{INTRODUCTION}

When working towards point-of-care systems, ease-ofuse and fast data processing become major focus points during device development. Measurement equipment that is now confined to the development of state-of-the-art novel biosensors in a laboratory environment, needs to become more reasonably priced, smaller and user-friendly to penetrate the consumer electronics market.

A first step towards portable biosensor readout systems was already taken with the development of a miniaturised impedance spectroscopy unit, based on the Analog Devices AD5933 gain/phase analyzer [1]. Though having achieved a small and cheap device, in the quest for a handheld system which might prove commercially viable, more attention is needed for the user-friendliness aspect. The newly developed handheld measurement device, shown in Fig. 1, operates fully stand-alone and is fine-tuned for electronic readout of bio- and biomimetic analytical sensors, e.g. DNA denaturation measurements or immunosensing [2, 3, 4].

Healthcare could benefit greatly from remote monitoring techniques [5]. When moving towards mobile healthcare applications, diagnosis time is reduced and cost efficiency can be greatly increased $[6,7]$. Therefore several communication protocols have been implemented in the device, which provide the end-user with convenient ways of measurement analysis and remote readout.

One promising line of research in biosensors is molecular imprinting [8, 9, 10, 11]. Molecularly Imprinted Polymers (MIPs) are synthetic biomimetic polymers with imprinted nano-cavities that form preferential binding sites for the desired target molecule. MIPs have already been proven to be extremely suited for the detection of various low molecular weight targets such as histamine [12, 13], Lnicotine [14] and adenine [15]. Readout of such sensors is commonly performed by impedance spectroscopy $[1,16]$ or gravimetric sensing $[15,17]$. The suitability of the newly developed handheld impedance analyser for biosensor readout will be verified based on several impedance spectroscopy measurements on L-nicotine MIP biosensors.

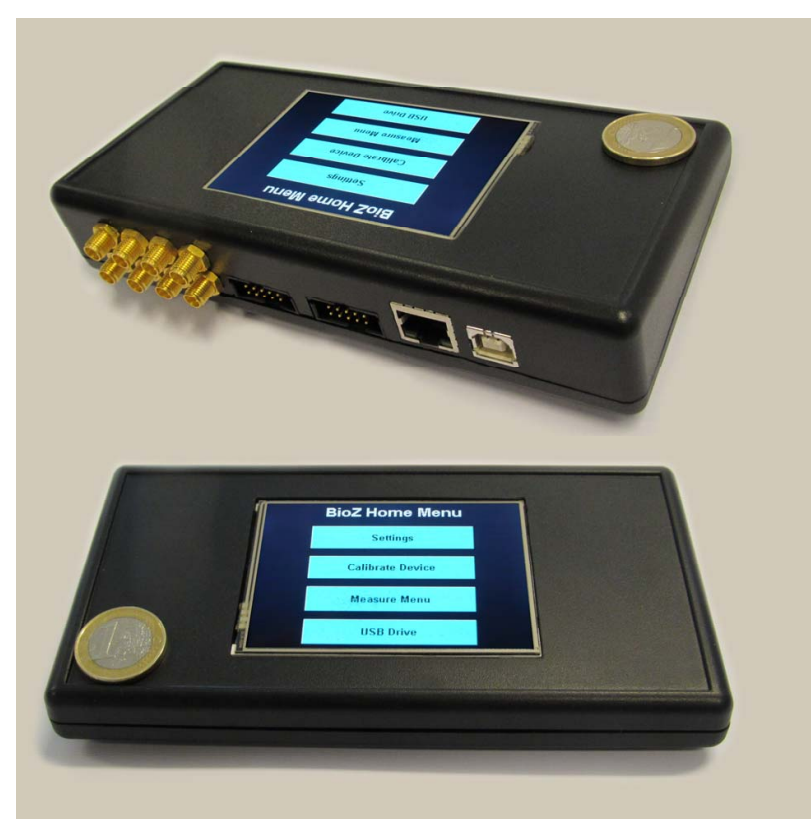

Figure 1. The developed point-of-care device, with coaxial sample connectors, Ethernet and USB port (top) and startup menu (bottom) visible. 


\section{MATERIALS AND METHODS}

As shown by the simplified block diagram illustrated in Fig. 2, a central microcontroller interfaces to different hardware blocks dedicated to the incorporated communication protocols. The impedance analysis subcircuit, based around the AD5933 chip, is shown in the upper part of the block diagram. This sub-circuit is completely controlled via an $\mathrm{I}^{2} \mathrm{C}$ bus. The use of separate bus lines for each hardware block eliminates interference while increasing speed. In subsequent paragraphs the different key hardware elements are discussed.

\section{A. Impedance spectroscopy}

Although the AD5933 IC is able to perform frequency sweeps over a fixed, narrow impedance range, peripheral circuitry was required to enable measurements in a broader impedance range and frequency spectrum. When operating on the internal oscillator of $16 \mathrm{MHz}$, the impedance chip is able to perform frequency sweeps from $3 \mathrm{kHz}$ to $100 \mathrm{kHz}$. A Maxim Integrated Products DS1077 IC was used as an external clock to generate clock frequencies in a range of $10 \mathrm{kHz}$ up to $66 \mathrm{MHz}$, lowering the AD5933 minimum excitation frequency to $10 \mathrm{~Hz}$. It should be noted that the upper frequency limit, $100 \mathrm{kHz}$, could not be altered.

To avoid any interference of the excitation signal on the biological measurements, the default excitation voltage of $2 \mathrm{~V}$ is attenuated to $60 \mathrm{mV}$ by means of a simple resistor based voltage divider. Any present DC offset voltage is removed by a series capacitor. The signal is buffered by means of a unity gain amplifier. The resulting current caused by the impedance of the sample is again converted to a voltage by means of an inverting OPAMP circuit with a set of switchable feedback resistors. Since the AD converter inside the AD5933 has a resolution of only 12 bits, the measurable impedance range would be very limited. By using 5 switchable feedback resistors in the I-V OPAMP circuit, the complete impedance range, set from $10 \Omega$ to $1 \mathrm{M} \Omega$, is divided into 5 sub ranges. Each sub range utilizes the 12 bits resolution of the impedance chip. The AD5933 is only able to analyze positive voltages, thus a Zener diode circuit is used to add a suitable DC offset of $1.6 \mathrm{~V}$.

While the device built around these principles, further documented in [1], is able to perform accurate impedance measurements over eight channels, it was found lacking in the field of user control and interfacing. Being fully dependent on a PC for power supply and system control, a communication bottleneck exists at the conversion from USB to the $\mathrm{I}^{2} \mathrm{C}$ bus controlling measurement hardware and peripheral circuitry inside the device. Furthermore, other processes running on the $\mathrm{PC}$ could slow down the measurement data collection. As the desirable impedance spectrum lies between $10 \mathrm{~Hz}$ to $100 \mathrm{kHz}$, several noise sources inside the PC could negatively interfere with the measurement signals. Nevertheless, this analyser provides a simple yet effective solution for measuring biosensors in lab setups or even basic field applications. Thus this measurement circuitry was used as a starting point for

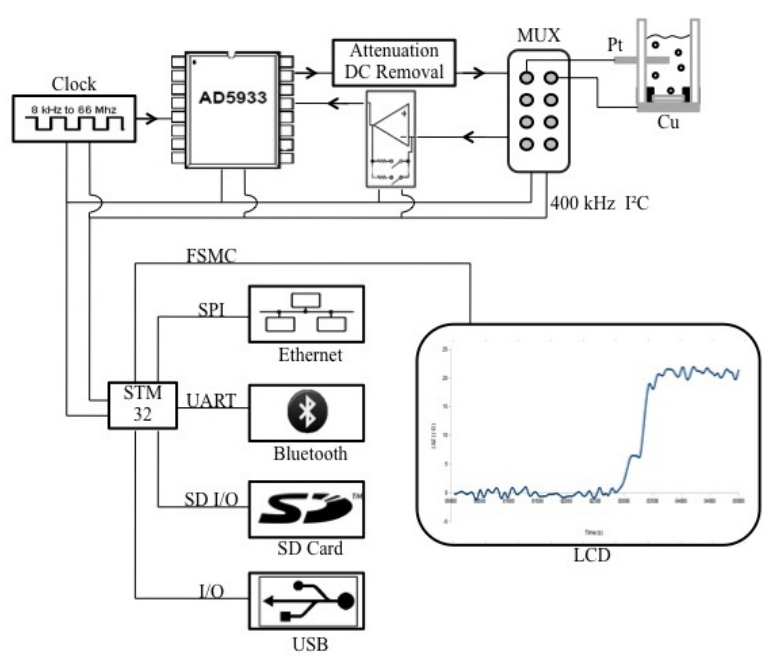

Figure 2. Simplified block diagram.

development of the current, more advanced point-of-care system.

\section{B. Embedded processing}

Driving towards smaller, faster and low cost applications embedded systems have become part of our everyday life. Because the device is designed to perform a couple specific tasks it is important that it performs those tasks with great performance and reliability. The requirements that helped to determine the right micro controller unit (MCU) were the need for at least $300 \mathrm{kB}$ of Flash and $30 \mathrm{kB}$ of RAM. These relatively demanding characteristics are mainly caused by the need for extensive data processing when analyzing the measurement results. Next to these there were also the requirements for several peripherals that will be discussed later on. The ARM Cortex-M processor is by now 8 years old and has achieved huge success during this time, e.g. in biomedical applications [18, 19]. The RISC instruction set makes it very suitable for embedded applications. The Cortex-M3 is the most mature member of the family and offers a very versatile range of products and proves to be the most suited core for our application [20].

The choice was made to implement the STM32F103VET6 manufactured by ST Microelectronics [21]. This MCU has $512 \mathrm{kB}$ of FLASH onboard combined with $64 \mathrm{kB}$ of RAM. Aside from that it has all the necessary protocols on board. Due to the complexity of the project a Real Time Operating System (RTOS) is a critical and necessary choice to guarantee proper task handling. A well designed RTOS is the Micrium $\mu \mathrm{C} / \mathrm{OS}-\mathrm{II}$ [22]. This is capable of low-cost priority-based pre-emptive multitasking. It is highly suitable for use in safety critical applications such as medical systems and has been ported to a large range of processors to allow easy implementation which benefits time-to-market. 


\section{User interfacing}

One tendency in devices currently being released or under development for (bio)medical applications is the incorporation of touch-screen displays. It has been shown that patients can easily adapt to touchscreen technology for fast diagnosis system [23]. Examples of such applications can be found in quality of life control for terminal patients $[24,25]$. An ILITEK ILI9320 a-Si TFT 262K color LCD screen with a resistive touchscreen layer was interfaced via the flexible static memory controller (FSMC) bus to the device. The Texas Instruments ADS7843 touchscreen controller is used to read out user input over the Serial Peripheral Interface(SPI) bus. This provides the users with full control over measurement settings and allows for results analysis in a graphical manner.

Many modern day commercial biosensor applications also implement TCP/IP protocol for remote monitoring $[26,27]$. This way, measurements can be monitored remotely over a standard local area network (LAN) or over the Internet. Aside from being a convenient feature in lab environments, consumer health could benefit largely from this advance in technology. Remote monitoring could mean a significant increase in patient security and comfort, while reducing diagnosis time [5]. An Ethernet communication circuit was implemented in the device to enable networkbased control. At the core of this sub-circuit lies the Microchip ENC28J60 Ethernet Controller [28]. This controller communicates with the previously discussed ARM Cortex M3 microcontroller that runs the webserver and generates the W3 compliant HTML website the user can visit via the local network. The interfacing with the microcontroller is achieved via a Serial Peripheral Interface (SPI) bus. Users can log in onto a user-defined passwordsecured website where, depending on pre-set user privileges, measurements can be monitored or access can be granted to control the device. Remote recalibration and configuration of measurement settings is possible as well.

With this implementation of both touchscreen and Ethernet module, direct device control and large-distance control are fully covered. It may however be desirable for certain applications to wirelessly monitor the measurements over a short distance. Bluetooth was chosen as the most suited protocol to serve this purpose. Aside from already being commonly used in wireless patient monitoring systems, this protocol was for example also used for crowd movement studies [29, 30]. An Amp'ed RF BT-23 miniature Bluetooth module with integrated antenna was interfaced to the central microcontroller by means of UART protocol [31]. This module supports the Bluetooth v2.1 Serial Port Profile (SPP) protocol at typical throughput of $1,5 \mathrm{Mbit} / \mathrm{s}$. The pairing password that is necessary to connect to the device can be changed by the user. The wireless exchanged data is secured with a 128 bit encryption key. Aside from these communication protocols for data transfer, the measurements can also be stored on an on-board Secure Digital (SD) card.
The RTOS hosts a file system module which allows the user to write or read data from the SD card from the device directly using its built in USB mass storage device driver, or by reading out the SD card directly using any type of system capable of reading FAT formatted SD cards.

\section{PCB layout}

When combining the analog/digital measurement circuitry with purely digital processing and interfacing, special care should be taken for noise and interference reduction. The choice was made to reduce interference by careful board-design. It is a generally accepted technique to split analog and digital power planes, bringing them together at analog-digital interfacing components. As the AD5933 impedance chip is the only component in the circuit which combines both analog and digital signals, this split power plane can easily be achieved. Furthermore, with the $\mathrm{I}^{2} \mathrm{C}$ bus being the only means of communication between the embedded processor circuitry and the impedance analysis sub-circuit, interference between these two circuits can be easily separated at board-layout level. Fig. 3 illustrates the designed printed circuit board (PCB).

One can clearly distinguish the different sub-circuits. The left part of the PCB contains the ARM microcontroller together with the peripheral IC's, including the Ethernet and Bluetooth controller. The right side contains the impedance measurement circuitry, further divided into an analog and digital part. A PCB stack-up was chosen where both top and bottom layer function as signal layers while the 2 inner layers are configured as power- and ground plane.
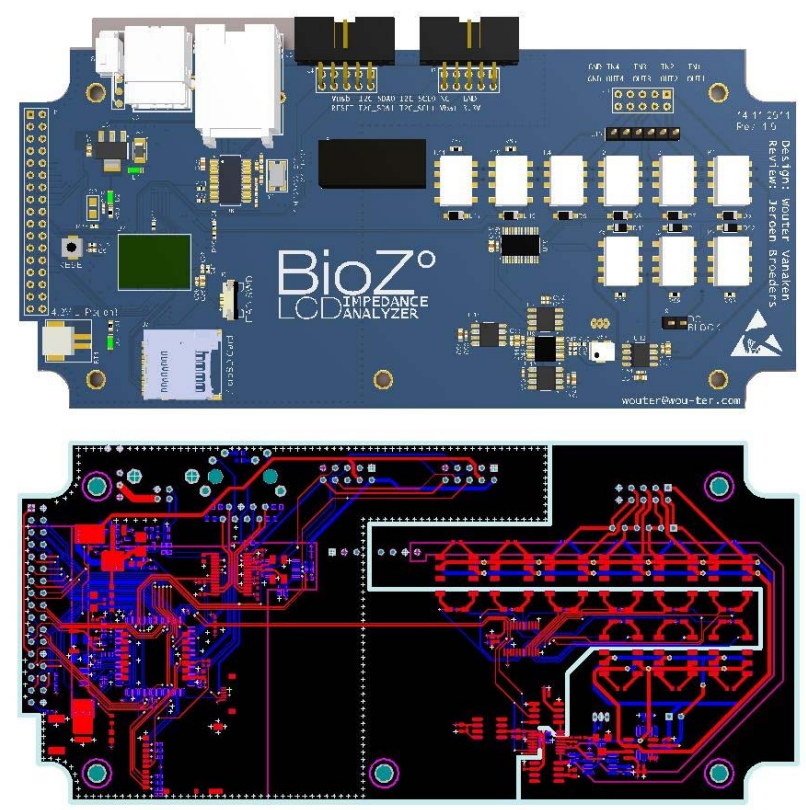

Figure 3. PCB design showcasing the separated power planes. 
Implementing this technique provides some important benefits. The power plane and adjacent ground plane are capacitively coupled, reducing the number of bypass capacitors needed on the board and providing better power supply filtering. Having a solid ground plane also eases grounding the components on the board preventing the creation of unintended grounding loops caused by traced grounds. It also allows signals to be routed in a micro-strip configuration.

The ground plane decreases ground impedance, and therefore ground noise, significantly. The adjacent power plane makes it easier to supply the required voltages right underneath the part so thick and long power traces can be avoided [32].

The device is enclosed in an ABS casing, also containing a rechargeable Lithium Polymer $950 \mathrm{mAh}$ battery as main power supply. Power consumption is rated at $100 \mathrm{~mA}$ for the impedance circuit and $130 \mathrm{~mA}$ for the microcontroller with peripheral circuitry. This indicates a battery life of about 4 hours, sufficient to perform rapid assessment with biological sensors.

\section{PERFORMANCE ANALYSIS}

As was addressed in the previous paragraph, the device should be able to perform measurements in a frequency spectrum between $10 \mathrm{~Hz}$ and $100 \mathrm{kHz}$ with sensorimpedance varying between $10 \Omega$ and $1 \mathrm{M} \Omega$. In order to test the performance of the newly developed unit, verification measurements were performed on passive components.

To gain insight in the accuracy of the device, a set of resistors from the E12 series were measured at a fixed frequency of $1 \mathrm{kHz}$. The value of each resistor was verified with a Keithley 2000 series digital tabletop multimeter, after which the deviation and phase shift was calculated. Figure 4 shows the deviation of the measured impedance and phase shift in respect to the resistor value.

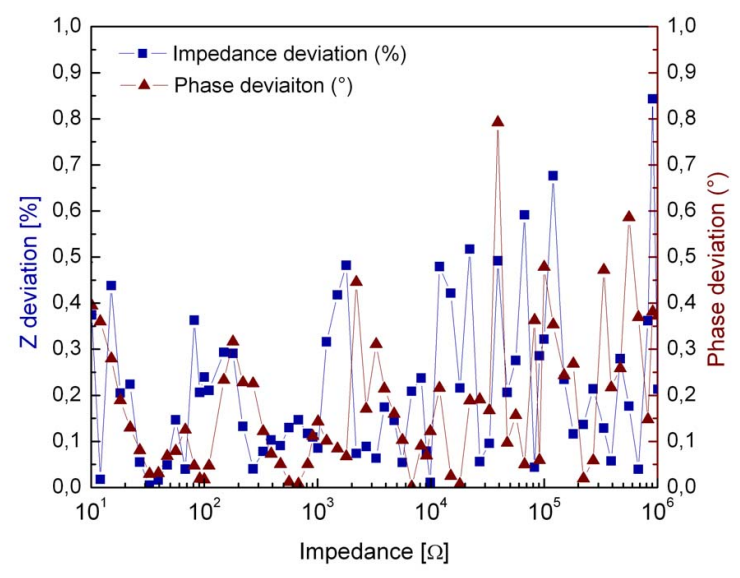

Figure 4. Deviation on a measured set of resistors.
The average measurement error on this set of resistors is $0,22 \%$, with a standard deviation of $0,18 \%$. The $95^{\text {th }}$ percentile lies at $0,59 \%$ and a maximum deviation of $0,84 \%$ can be seen at $900 \mathrm{k} \Omega$. When looking at the impedimetric response of biosensors to target molecule detection, typical values can be seen in the range of several percent, even for detection of concentrations just above the detection limit of the sensor itself $[1,2,4]$. Therefore the device accuracy lies well within specifications for accurate biosensor readout.

Typical biosensor layouts consist of a sensing layer, immobilized on a (semi)conducting substrate, which is placed in a liquid analyte. Impedance spectroscopy can be performed by using the substrate as working electrode and a conductor placed in the liquid as counter electrode. This way the substrate, liquid and counter electrode can be seen as purely resistive elements. The interface between the sensing layer and liquid behaves like a parallel resistorcapacitor (RC) circuit. Equivalent circuits can thus be calculated consisting of the parallel RC circuit with a series resistance.

An equivalent circuit was set up similar to the liquid cell commonly used for biosensor applications. The circuit consists of a $3 \mathrm{nF}$ capacitor in parallel with a $82 \mathrm{k} \Omega$ resistor to simulate the interfacial double-layer. A $330 \Omega$ resistor is placed in series. The circuit was measured with the newly developed unit as well as with a Hewlett Packard (HP) 4194a impedance/gain-phase analyzer used as a reference [33]. Fig. 5 illustrates the Bode plots for the equivalent circuit, measured with both devices from $100 \mathrm{~Hz}$ to $100 \mathrm{kHz}$ with 10 subdivisions per decade.

It should be note that the HP4194a is not able to perform impedance spectroscopy below $100 \mathrm{~Hz}$, therefore the decade of $10 \mathrm{~Hz}$ to $100 \mathrm{~Hz}$ was also not plotted for the newly developed device. These Bode plots indicate correct functioning of the unit over the desired frequency spectrum, although an increase in impedance deviation between both devices can be seen towards $10 \mathrm{kHz}$.

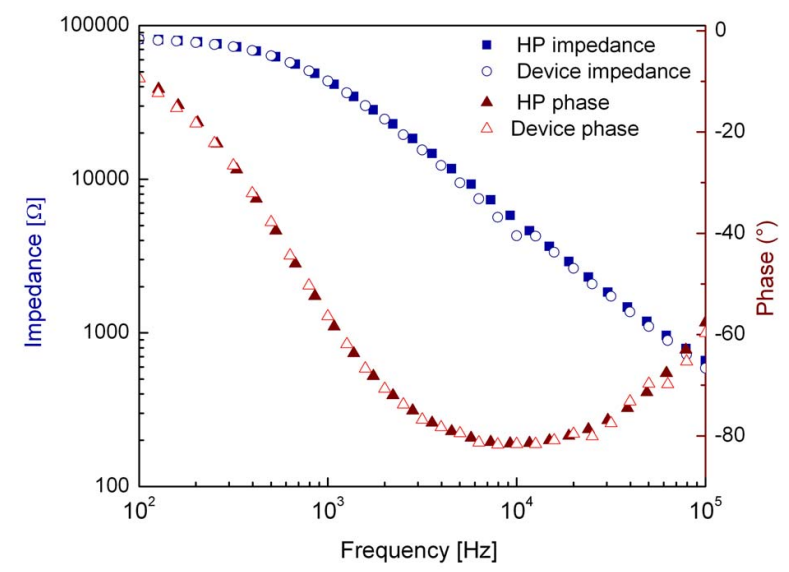

Figure 5. Bodeplots for the equivalent circuit. 
As was mentioned in the second paragraph, the complete impedance range is divided into five sub ranges by means of a set of relay-switchable inverting OPAMP feedback resistors. The deviation seen around $10 \mathrm{kHz}$ is most likely the last point in a certain sub-range, after which a feedback resistor is switched resulting again in a low deviation.

\section{BIO ANALYTICAL MEASUREMENTS}

\section{A. Sensor and measurement setup}

The biosensor chosen for the verification of this impedance analyzer is a biomimetic L-nicotine sensor [14]. A molecularly imprinted polymer (MIP) layer is deposited on top of a $1 \mathrm{~cm}^{2}$ thin titanium (Ti) plate. The main advantage of using Ti surfaces is its naturally occurring thin oxide surface layer, which is required for good deposition and surface adherence of the MIP layer. The Ti transducer surfaces needed to be prepared to enable anchoring of the sensor layer. This was done by silanizing the surfaces, following the protocol described in [17].

For the preparation of the MIP itself, a mixture of $12.5 \mathrm{mmol}$ methacrylic acid (MAA), $25.2 \mathrm{mmol}$ ethylene glycol dimethacrylate (EGDM) and $1.5 \mathrm{mmol}$ azobisisobutyronitrile (AIBN) was dissolved in $7 \mathrm{ml}$ toluene together with the template molecule L-nicotine $(6.41 \mathrm{mmol})$. These solvents were of analytical grade and used without purification. All products were purchased from Acros Organics, except for the AIBN, which was purchased from Fluka.

To exclude oxygen in the mixture, it was de-gassed for 10 min with $\mathrm{N}_{2}$. This mixture was introduced onto the $\mathrm{Ti}$ surface, where it was heated at $65^{\circ} \mathrm{C}$ for 10 minutes to induce the polymerization. After polymerization the solid MIP was extensively washed using repeated flushing with ethanol.

A measurement cell was constructed and interfaced to the impedance analyzer to enable readout of this sensor. In lab setups several peripheral devices can be used to give complete control over the sensor's environment issues. It is for example common to control the temperature of the sensor to mimic in-vivo circumstances [12], or to use pumping systems as a way to flow in different concentrations of analyte [34]. Since the developed unit features a step in the development towards point-of-care systems, the choice was made to keep the sensor setup as simple as possible in these measurements. A Teflon liquid reservoir was constructed with a copper lid. The substrate with the MIP sensor layer was attached to this lid and mounted into the cell. The cell was then filled with a $1 \mathrm{x}$ phosphate buffer saline (PBS) solution at $\mathrm{pH} \mathrm{7,} \mathrm{after} \mathrm{which}$ different concentrations of the target molecule were added. PBS buffer is used to keep $\mathrm{pH}$ constant while simultaneously mimicking the ionic strength and $\mathrm{pH}$ of human body liquids. Impedance measurements were continuously performed by using the substrate with sensor layer as working electrode and a chemically inert platinum wire as counter electrode positioned several millimeter above the sample in the cell.

\section{B. Nicotine sensing}

Biomedical measurement data can be displayed on the LCD screen in the form of time-based detection curves, as shown in Fig. 6. A measurement cell was filled with pure PBS solution ( $\mathrm{pH} \mathrm{7)}$ and then subjected to increases in Lnicotine concentration. As can be seen in Fig. 6, the $10 \mathrm{nM}$ L-nicotine concentration went undetected, while a clear impedance change was measured for $20 \mathrm{nM}$. Detection sensitivity and upper and lower limits are dependent on the quality of the biomimetic MIP layer. The short, small dips in impedance at concentration changes are the result of usergenerated disturbances in the system upon addition of Lnicotine.

Such time-dependent graphs are generated from monitoring the measured Bode plots at a fixed, userselectable frequency. Fig. 7. shows these Bode plots for the initial environment of pure PBS and an analyte consisting of $100 \mathrm{nM}$ of L-nicotine dissolved in PBS. One can clearly distinguish dropping impedance over increasing frequency for both analytes, due to a capacitive component. This can also be seen in the phase, where a maximum phase shift of $-60^{\circ}$ is measured. This capacitance is caused by the interface between sensor layer and analyte. Furthermore, in the upper frequencies a constant impedance is seen in combination with a phase shift approaching zero. This is most likely due to resistivity of the analyte, substrate and electrodes. It should be noted that a resistive component, parallel with the capacitance, is commonly present in electrochemical cells. The presence of this resistivity can be seen by the decreasing phase at low frequencies. This component is however not of interest when analyzing the response of the biomimetic sensor.

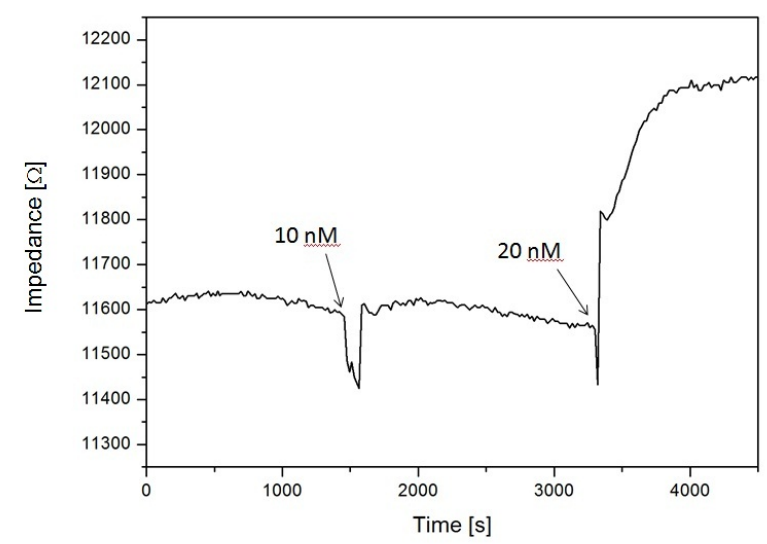

Figure 6. Impedance response to the addition of $10 \mathrm{nM}$ and $20 \mathrm{nM}$ of L-nicotine. 

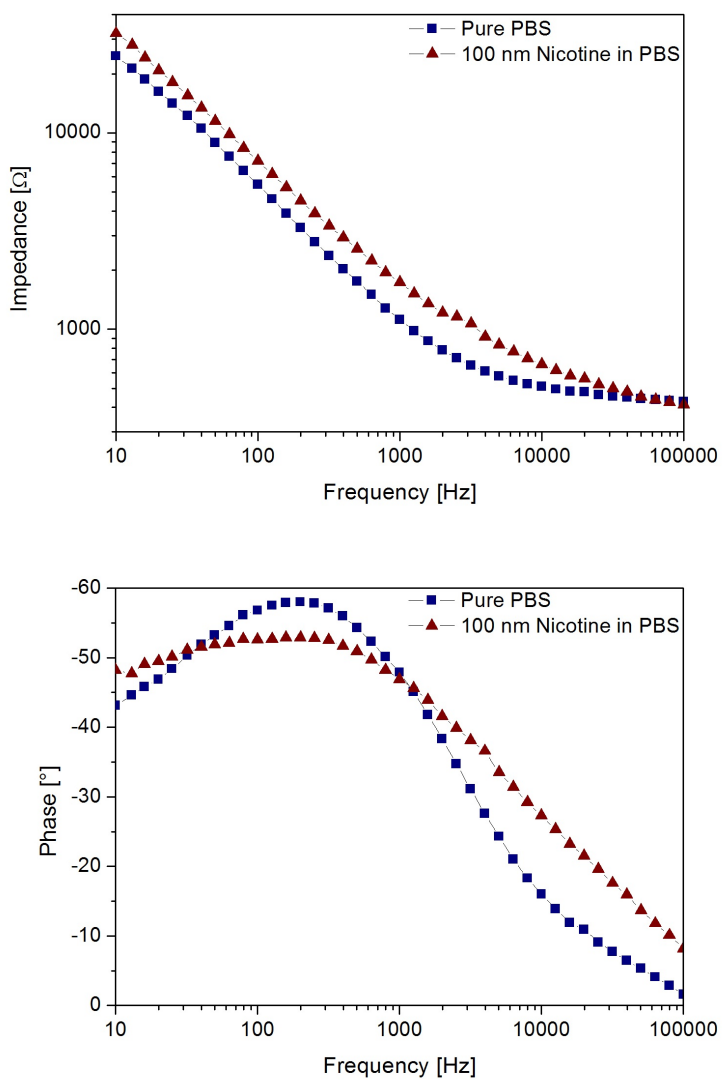

Figure 7. Bodeplots at 1x PBS and $100 \mathrm{nM}$ nicotine concentration in PBS.

Impedimetric response upon detection of target molecules in a MIP layer can be most significantly seen in the interfacial capacitance $[1,14]$. It was therefore chosen to analyze the sensor detection at the frequency corresponding with the highest phase shift. As can be seen in these Bode plots, this points corresponds with a frequency of $200 \mathrm{~Hz}$. The capacitance was calculated at this frequency for both analytes using the imaginary impedance component. This capacitance drops from $270 \mathrm{nF}$, at pure PBS, to $218 \mathrm{nF}$ for the $100 \mathrm{nM}$ L-nicotine concentration. The user can choose to have this capacitance plotted on the display, which could fasten analysis. In practical applications, e.g. urine analysis, L-nicotine concentrations above $100 \mathrm{nM}$ need to be detected [14]. It becomes clear that the device accuracy is high enough to measure sensor response in practical setups.

\section{DISCUSSION}

A newly developed, completely stand-alone, miniaturised impedance analyzer is presented. Aside from incorporating circuitry specifically fine-tuned for bio-analytical impedance spectroscopy, the device has several communication protocols on board for convenient monitoring of measurements. These protocols include Bluetooth for wireless readout and Ethernet for network monitoring. Furthermore, a touchscreen display greatly elevated the usability of the device, making it applicable in consumer setups.

The accuracy of the system was tested on passive components. To do so, a set of resistors ranging from $10 \Omega$ to $1 \mathrm{M} \Omega$ was measured, with which an average accuracy of $0,22 \%$ was achieved. Furthermore, impedance sweeps were performed on an equivalent circuit of a previously discussed measurement cell. These measurements were compared with a high-end HP impedance analyzer. Results indicate correct functioning of the unit in the desired frequency spectrum.

To demonstrate the usability on biosensors, a synthetic molecular imprinted polymer (MIP) layer, suited for detection of L-nicotine was measured. The increase of impedance upon addition of L-nicotine could be clearly monitored. Since this impedance change is caused by changes in interfacial capacitance, the user can choose to have the capacitance changes of the sensor layer displayed on the device.

Aside from becoming a vital part in lab setups, this unit creates a bridge between technologies incorporated in common day smartphones and specialised measurement equipment. This way a basis is set for commercially available, impedance based biosensor devices. Further research will focus on increasing the applicability of different types of biosensors in consumer applications. Practical usability of the device in point-of-care settings will also be further evaluated, while keeping a close eye on the aspects of user-friendliness and security.

\section{ACKNOWLEDGMENT}

The authors would like to thank the special research funds of Hasselt University and Xios University College for the necessary financial resources. Furthermore we owe thanks to Jan Mertens, Lieven De Winter, Johnny Baccus and Johan Soogen for technical support.

\section{REFERENCES}

[1] J. Broeders et al., "Miniaturized eight-channel impedance spectroscopy unit as sensor platform for biosensor applications", Phys. Stat. Sol. A, vol. 208, pp. 1357-1363, 2011

[2] B. van Grinsven et al., "Rapid assessment of the stability of DNA duplexes by impedimetric real-time monitoring of chemically induced denaturation", Lab Chip, vol. 11, pp. 1656-1663, 2011.

[3] B. van Grinsven et al., "Heat-tranfer resistance at solid-liquid interfaces: a tool for the detection ofsingle nucleotide polymorphisms in DNA", ACS Nano, in press.

[4] P. Cooreman et al., "Impedimetric immunosensors based on the conjugated polymer PPV”, Bios. Bioelectron., vol. 20, pp. 2151-2156, 2005.

[5] P. St-Louis, "Status of point-of-care testing: promise, realities and possibilities", Clin. Biochem., vol 33, pp. 427-440, 2000. 
[6] R.W.T. Ng, A. Laurent, C. Sie Boo, "Ultra low power SOC for portable health monitoring platforms", Proc. IEEE Integrated Circuits (ISIC) Symp., IEEE press, Dec. 2011.

[7] W. Siong, J.C.M. Teo, W. T. Ang; S. Viswanathan, C. K. Tham, "Experiences on developing SOA based mobile healthcare services", Proc. IEEE Services Computing Conf., IEEE press, Dec. 2009.

[8] C. Alexander et al., "Molecular imprinting science and technology: a servey of the literature for the years up to and including 2003", Mol. Recognit., vol. 19, pp. 106-180, 2006

[9] D. R. Kruscio, N. A. Peppas, "Critical review and perspective of macromolecularly imperinted polymers", Acta Biomat., vol. 8, pp. 461-473, 2012

[10] C. A. Barrios, C. Zhenhe, F. Navarro-Villoslado, D. Lopez-Romero, M. C. Moreno-Bondi, "Molecularly imprinted polymer diffraction grating as label-free optical bio(mimetic)sensor", Biosens. Bioelectron., vol. 26, pp. 2801-2804, 2011.

[11] S.-C. Huang et al., "A microfluidic chip integrated with molecular imprinting polymer for surface plasmon resonance detection", Proc IEEE Solid-State Sensors, Actuators and Microsystems, IEEE press, June 2005.

[12] E. Bongaers et al., "A mip-based biomimetic sensor for the impedimetric detection of histamine in different $\mathrm{pH}$ environments", Phys. Stat. Sol. A, vol. 207, pp. 837-843, 2010.

[13] F. Horemans et al., "Mip-based sensor platforms for the detection of histamine in the nano- and micromolar range in aqueous media", Sens. Actuators B, vol. 148, pp. 392-398, 2010.

[14] R. Thoelen et al., "A mip-based impedimetric sensor for the detection of low-mw molecules", Biosens. Bioelectron., vol. 25, pp. 913-918, 2008.

[15] A. Pietrzyk et al., "Molecularly imprinted polymer (mip) based piezoelectric microgravimetric chemosensor for selective determination of adinine", Biosens. Bioelectron., vol. 25, pp. 25222529, 2010.

[16] B. Khadro, C. Sanglar, A. Bonhomme, A. Errachid and N. JaffrezicRenault, "Molecular imprinted polymers (MIP) based electrochemical sensor for detection of urea and creatinine", Proc. Engineering, vol. 5, pp. 371-374, 2010.

[17] D. Croux et al., "Development of multichannel quartz crystal microbalances for MIP-based biosensing", Phys. Stat. Sol. A, vol. 209, pp. 892-899, 2012

[18] S. Ravindran, R. Cole, "Low complexity algorithms for heart rate and epileptic seizure detection", Proc. IEEE Applied Sciences in Biomedical and Communication Technologies, IEEE press, Nov. 2009.

[19] S. R. Sridhara, "Microwatt embedded processor platform for medical system-on-chip applications", Proc. IEEE Symp. VLSI Circuits (VLSIC), IEEE press, June 2010.

[20] J. Yiu, "The definitive guide to the ARM Cortex M3", Newness, 2009.

[21] STMicroelectronics, "STM32F103XC, STM32F103XD, STM32F103XE datasheet", 2011.

[22] Micrium, " $\mu \mathrm{C} / \mathrm{OS}-\mathrm{II}$ Real-time operating system datasheet", 2011

[23] A. M. Piper, J. D. Hollan, "Supporting medical communication for older patients with a shared touchscreen computer", Int. Journ. Of Medical Informatics, in press.

[24] P. Thekkumpurath, C. Venkateswaran, M. Kumar, A. Newsham, M. I. Benntt, "Screening for psychological distress in palliative care: performance of touchscreen questionnaires compared with semistructured psychiatric interview", Journ. Of Pain and Sympt. Manage., vol. 38, pp. 597-605, 2009.

[25] E. H. Lee, "Touch-screen computerized quality-of-life assessment for patients with cancer", Asian Nursing Research, vol. 3, pp. 41-48, 2009.

[26] Nova Biomedical Corporation, "StatSensor specifications", 2010.

[27] Ohmx Corporation, "Handheld Biosensor Reader datasheet", 2006.
[28] Microchip Technology Inc., "ENC28J60 datasheet", 2008.

[29] S. Pandey, W. Voorsluys, S. Niu, A. Khandoker and R. Buyya, "An autonomic cloud environment for hosting ECG data analysis services", Fut. Gen. Comp. Sys., vol. 28, pp. 147-158, 2012.

[30] M. Versichele, T. Neutens, M. Delafontaine and N. Van de Weghe, "The use of Bluetooth for analysing spatiotemporal dynamics of human movement at mass events: a case study of the Ghent Festivities", Appl. Geogr., vol. 32, pp. 108-220, 2012.

[31] Amp'ed RF Technology Coorporation, "BT23 datasheet", 2011.

[32] A. Riazi, "PC board stack up reference", Printed Circuit Design \& Fab, October 2008 .

[33] Agilent Technologies, "4194A Impedance/Gain-Phase Analyzer datasheet", 1986

[34] B. van Grinsven et al., "Customized impedance spectroscopy device as possible sensor platform for biosensor applications", Phys. Stat. Sol. A, vol. 207, pp. 919-923, 2010. 